\title{
VALENZA E INSEGNAMENTO DELLA LINGUA STRANIERA*
}

\author{
Ignazio Mauro MIRTO
}

Università di Palermo

\begin{abstract}
En): This work deals with valency, a key syntactic notion whose chemistry-inspired label was established by Lucien Tesnière (1959), one of the most well-known contributors to the Prague Linguistic Circle. We will concentrate on two questions: (a) to what extent does valency play a role in Second Language Learning (SLL)?; and (b) what role should the use of valency play in language teaching practices? This paper will provide answers to these questions and propose reasons for motivating the use of valency in SLL.
\end{abstract}

Keywords: Second language learning; transfer; L1-L2 valency mismatches; noticing

Mots-clés : Didactique des langues étrangères ; interférence ; différences valencielles en L1L2 ; noticing

\section{Introduzione}

Con l'etichetta di 'valenza' o, più recentemente, con 'sottocategorizzazione' e 'struttura argomentale', in letteratura si fa oggi riferimento a quella complessa materia che riguarda, da un lato, il numero di elementi, gli argomenti, che un predicato aggrega e, dall'altro, la proprietà di stabilire col predicato un obbligatorio rapporto di tipo sintagmatico. Uno sguardo sistematico e moderno in questo àmbito ${ }^{1}$, limitato ai verbi ma con effetti importanti per la linguistica, lo si deve a Lucien TESNIÈRE (1959), che fu contributor francese del circolo linguistico praghese (v. HAJIČOVÁ, 2006: 65). Allo studioso va attribuito il fortunato termine 'valenza', ispirato dalla chimica.

Questo contributo verte sul ruolo che il concetto di valenza svolge e può svolgere nella riflessione teorica elaborata dagli studiosi di linguistica applicata all'insegnamento / apprendimento delle lingue straniere (LS). Si vuole anche fare cenno alle ricadute pratiche di tale riflessione, all'utilità che la valenza potrebbe avere nei testi didattici destinati all'apprendimento di una LS e a quanto essa condizioni e guidi la scrittura di programmi specifici, tanto nei livelli successivi alla scuola primaria quanto all'università. Intendiamo sottolineare come gli studi teorici nell'àmbito della valenza verbale possano risultare utili nel campo dell'insegnamento delle lingue, un interesse già vivo, come fa notare FRIED (1972: 6) nella citazione che segue, in Vilém Mathesius, il fondatore del Circolo Linguistico di Praga, il quale nel 1929, in un congresso destinato a insegnanti di grammatica, ebbe ad affermare che: «Every truly great idea must find practical application and have practical consequences ${ }^{2}$.

\footnotetext{
${ }^{*}$ L'autore ringrazia i due relecteurs per i loro commenti.

${ }^{1}$ La nozione è ovviamente preceduta da secoli di riflessione, in particolare nella logica classica (v. la voce 'Predicato' nel Dizionario di filosofia di Nicola AbBagnano, 1971: 691). Alcuni cenni sui precursori della nozione di valenza sono reperibili in RIEGER (2003).

${ }^{2}$ Secondo VACHEK (1972: 11): «the Prague group has always been noted for its systematic effort to derive practical consequences from its theoretical findings».

Echo des études romanes XI/1, $2015 \quad-137$ -

ISSN: 1804-8358 (Online)
} 


\section{Transfer ${ }^{3}$}

La nostra convinzione riguardo alla valenza è che nell' apprendimento guidato di una qualsiasi LS essa sia centrale e per nessun motivo trascurabile. Una non marginale quantità di errori prodotti dagli apprendenti pare infatti riconducibile, soprattutto nelle prime fasi, a transfer negativo generato da differenze valenziali tra il predicato della lingua madre (LM) che il discente ha in mente e il predicato che egli intende utilizzare per la resa in LS. Si tratta di itinerari traduttivi che l'apprendente mette in atto il più delle volte in modo del tutto inconsapevole. Come esempio di transfer negativo di questo tipo valga quello discusso e motivato da ROSEN (1991). A studenti americani di livello universitario, al primo anno di studio della lingua italiana, tale transfer fa produrre frasi mal costruite come (1):

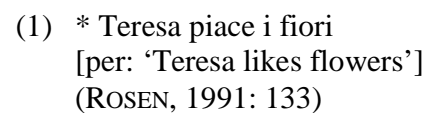

L'errore che questi apprendenti commettono è facilmente spiegabile tenendo conto delle differenze nelle valenze dell'italiano piacere e dell'inglese to like. Si tratta di verbi denominati 'psicologici'. Il primo è intransitivo. Nella corretta $A$ Teresa piacciono i fiori, l'argomento con il ruolo semantico di Stimolo è codificato come Soggetto (posposto, con ordine lineare marcato rispetto a quello canonico in italiano ${ }^{4}$ ), mentre l'argomento con il ruolo semantico di Esperiente è realizzato come Oggetto Indiretto. Il secondo è invece transitivo, con l'Esperiente come Soggetto (dunque in posizione pre-verbale) e lo Stimolo come Oggetto Diretto.

In (1), l'apprendente fa sì uso di parole italiane, ma è come se, per costruire la proposizione, con esse riempisse le 'caselle' di una struttura inglese. Pur se esposto a dati della LS provenienti dall'insegnante e da libri di testo, nella early grammar del discente la struttura inglese resta dominante ${ }^{5}$. Si tratta di un fenomeno che qualsiasi docente di lingua straniera deve aver osservato: nei primi passi dell'apprendimento di una LS l'ordine delle parole della LM esercita una forte influenza sulla produzione in LS.

\section{Il concetto di valenza nei testi}

A fronte della rilevanza che a nostro avviso le valenze hanno per gli apprendenti di una LS, una domanda cui bisogna rispondere è la seguente: che spazio occupa la nozione in linguistica applicata e nei testi didattici? Una prima

\footnotetext{
${ }^{3}$ Preferiamo qui utilizzare il termine 'transfer' piuttosto che 'interferenza', che è di significato più ampio e per questo più soggetto ad ambiguità.

${ }^{4}$ La posizione post-verbale del Soggetto, che ha tratto [- Animato], potrebbe essere collegata alla presenza dell'Oggetto Indiretto [+ Umano], più in alto nella gerarchia di animatezza di CoMRIE (1981). Sulla scorta di studi precedenti, e per via di alcune proprietà di piacere, in una teoria multistratale RosEN (1991: 136-138) analizza il verbo come inizialmente transitivo. L'inaccusatività finale dipenderebbe da una 'valenza estesa' (DAvIES e DUBINSKY, 1991).

${ }^{5}$ È opportuno ricordare la latent language structure, un arrangement (sistemazione o messa in ordine) che SELINKER (1972: 175) appaia alla latent psychological structure di LENNEBERG (1967).
} 
impressione la fornisce un'osservazione di ALLERTON (2006: 301) nella Encyclopedia of Language and Linguistics, alla voce 'Valency grammar' (corsivo di chi scrive):

Valency is thus to be seen as the capacity a verb (or noun, etc.) has for combining with particular patterns of other sentence constituents [...] Such information is vital for language learners but was never adequately presented in traditional grammars and dictionaries.

Pur se riferite soltanto a grammatiche e dizionari, le parole di Allerton dicono già della insufficiente attenzione prestata alla valenza ${ }^{6}$. Del resto, tanto il metodo comunicativo quanto l'approccio da tempo più comune, quello funzionalenozionale, non sembrano favorirla per il ruolo tutto sommato marginale attribuito all'accuratezza grammaticale, a differenza di quanto accadeva nel metodo contrastivo $^{7}$, in cui si avvertivano riverberi del metodo grammaticale-traduttivo.

Ma non sono soltanto grammatiche e dizionari bilingui a trascurare la nozione di valenza. La situazione pare infatti identica anche sul versante più teorico, cioè nella ricerca svolta in linguistica applicata. In un volume intitolato The Acquisition of Second Language Syntax (BRAIDI, 1999), per esempio, ci si attenderebbe che la valenza, di capitale importanza in sintassi, svolgesse un ruolo centrale. Ma, scorrendo l'indice analitico del volume, questa aspettativa è presto delusa, come accade anche con la voluminosa sintesi (824 pagine) compiuta da Rod ELLIS in The Study of Second Language Acquisition (1994). Non va meglio sul fronte di dizionari ed enciclopedie destinati a chi si interessa di linguistica applicata. In The Dictionary of Applied Linguistics (JOHNSON e JOHNSON, 1998) si trova appena syntactic frame, non come voce specifica del dizionario, ma come singola occorrenza registrata nell'indice analitico. In tre voci della Encyclopedia of Language and Linguistics che sviluppano nozioni di linguistica applicata, la parola 'valenza' non ricorre mai (BROwN, 2006, 12.353 pagine) ${ }^{8}$.

\section{L'influenza della LM sulla LS}

Le valenze, verbali e non, sono solo una delle possibili fonti di errori in LS dovuti a influenze della LM. MITCHELL e MYLES (1998: 14, 112-117) riferiscono di uno studio longitudinale condotto dalla European Science Foundation su dieci coppie di lingue, italiano incluso (appaiata a inglese o tedesco), che le autrici riassumono con queste parole (1998: 14, corsivo di chi scrive):

\footnotetext{
${ }^{6}$ Nella parte conclusiva del contributo, tuttavia, l'autore fa riferimento a ricerche e iniziative tendenti a colmare questa lacuna. A suo avviso, però, il più delle volte esse rimangono destinate a specialisti del settore. In questo senso, vanno segnalati Dicovalence 2, una risorsa informatica realizzata da Karel Van den Eynde e Piet Mertens, che registra le valenze di più di 3.700 verbi semplici del francese, e il Dizionario Italiano Sabatini Coletti (SANSONI, 2008).

${ }^{7}$ Fra i primi a scrivere di Contrastive Analysis viene spesso citato Charles Fries (v. LADO, 1957). FRIED (1972: 6-7) fa tuttavia notare che Mathesius e il gruppo di Praga erano già soliti fare uso di comparazioni tra due lingue, fatto che induce a ritenerli precursori.

${ }^{8}$ Si tratta di: Second and Foreign Language Learning and Teaching di P. SKEHAN; Second Language Acquisition of Phonology, Morphology, and Syntax di R. Hawkins e C. LozAno; e Language Teaching Traditions: Second Language di D. MusuMECI.
} 
These ESF researchers argue that the early grammars produced by learners in their multilingual study show little trace of L1 influence.

Percentuali riguardanti l'influenza della LM sulla LS sono fornite anche da altri ricercatori. Sempre secondo MitCHELL e MYLES (1998: 30), si va dal 3\% al 51\%, mentre per BETTONI (2001: 119) gli errori riconducibili alla LM vanno dal $3 \%$ al $15 \%$. Rimane stabile la percentuale più bassa, come si vede, mentre varia di molto quella più alta, fino a far dubitare o delle percentuali stesse o della comparabilità delle metodologie utilizzate. I lavori non dichiarano in che misura gli errori dovuti a differenze valenziali incidano nelle percentuali.

Si esaminino ora altri casi come (1) per la coppia italiano LM - inglese LS, problematica in glottodidattica proprio per le numerose differenze valenziali tra $\mathrm{i}$ verbi delle due lingue, con l'ultima che, come in (1), predilige più frequentemente la transitività. Con una preparazione sporadica, non sistematica, non esplicita, e affidata solo a occasionali iniziative dell'insegnante, come dovrebbe l'apprendente affrontare le differenze in (2), (3) e (4) ${ }^{9}$ ?

(2) a. Everybody contributed to the debate

b. They contributed clothing for the relief of the poor

(3) a. You indulge in women and wine

b. Don't indulge your children

(4) a. I entered into his confidence

b. I entered the house

Gli esempi nelle frasi (a) presentano i verbi contribute, indulge e enter con valenze comparabili a quelle dei verbi italiani 'contribuire', 'indulgere' e 'entrare'. In questi casi i verbi in LS e LM si somigliano e hanno significati non troppo discosti da quelli in inglese: 'Tutti contribuirono al dibattito', 'Indulgi a donne e vino', 'Entrai nelle sue confidenze'. Che dire però delle frasi (b)? In esse i verbi vengono utilizzati transitivamente ${ }^{10}$, con differenze semantiche per niente trascurabili, più accentuate nel caso di indulge ${ }^{11}$. Doppie valenze come queste sono frequenti.

Le frasi in (5) illustrano un caso parallelo a (1): a un verbo transitivo in inglese ne corrisponde uno intransitivo in italiano:

\footnotetext{
${ }^{9}$ Per reperire le coppie di frasi ci siamo avvalsi dei seguenti dizionari: Il Grande Inglese 2008, di Fernando PICCHI (Hoepli, 2007); Dizionario inglese italiano - italiano inglese, di Giuseppe RAGAZZINI (Zanichelli, 1967).

${ }^{10} \mathrm{~A}$ variare, tra le frasi inglesi e quelle italiane, è talvolta l'abbinamento tra ruoli sintattici e ruoli semantici. Il ruolo semantico di Locativo del verbo 'entrare', ad esempio, in (4b) è abbinato a un Oggetto Diretto, realizzato con un sintagma nominale, mentre in italiano esso necessita di un ruolo sintattico che impone un sintagma preposizionale. Un metodo che sfruttasse le differenze valenziali potrebbe giovarsi della familiarità che l'apprendente ha già in LM: universalmente, un ruolo semantico è infatti codificabile con ruoli sintattici diversi, come accade ad esempio con l'Oggetto Diretto di una frase attiva quando questa viene volta al passivo.

${ }^{11}$ Nonostante la somiglianza dei significanti indulge e 'indulgere' (più nella forma scritta che in quella orale), il caso in (3b) differisce da (2b) e (4b) perché cambiano radicalmente i ruoli semantici e quindi il significato.
} 
(5) a. He incurred a penalty

b. Egli è incorso in una pena

Così, finché il transfer è positivo, cioè con valenze e ruoli semantici sostanzialmente identici, come nelle frasi (a) di (2), (3) e (4), non si presenta alcuna necessità di spiegazione da parte dell'insegnante di LS. Quando, però, le valenze dei verbi di LM e LS non sono comparabili e il transfer si prospetta negativo, un'istruzione esplicita diventa «vitale», come scrive Allerton. Senza tale informazione, l'apprendente pare destinato a commettere errori. Se poi è oltre il cosiddetto "periodo critico' ${ }^{12}$, è facile, a nostro avviso, che le somiglianze tra il significante del verbo italiano e uno della LS, come in (2), (3) e (4), lo inducano a trarre indebite conclusioni (false equivalenze, parallelamente ai false friends) tanto sulla struttura argomentale quanto sul significato.

Il raffronto qui proposto tra valenze in LM e in LS non costituisce affatto un tentativo di riesumazione della cosiddetta Contrastive Analysis, abbandonata per buoni motivi, fra i quali la natura troppo meccanica e ripetitiva degli esercizi e il peso eccessivo sulla memoria dei discenti. Detrattori della teoria hanno addirittura affermato che gli stessi presupposti della Contrastive Analysis si rivelano falsi. Ad esempio, LARSEN-FREEMAN e LONG (2014 [1991]: 54) hanno scritto (e sa di paradosso) che «the French sentences that correspond literally to their English equivalents are not necessarily the easiest to learn». Una riflessione può gettare un'ombra di dubbio su tale asserzione. Una volta che lo studente di LS fa propria, non necessariamente in modo consapevole, l'esistenza di differenze valenziali tra $i$ verbi di LM e quelli di LS, può successivamente insorgere il noto problema della sovrageneralizzazione. Andrebbe indagata, allora, la possibilità che sia quest'ultima a influire sulle difficoltà di apprendimento di cui parlano LarsenFreeman e Long.

Difficoltà paragonabili si osservano anche in diglossia, come mostrano gli esempi seguenti:

(6) Lo esci il pane dal frigorifero?

(7) Entra la macchina per favore

Frasi come (6) e (7) sono comunissime nell'italiano regionale di Sicilia e provengono chiaramente dalle altrettanto comuni valenze transitive dei verbi siciliani nèsciri 'uscire' (U niscisti u pani ru frigoriferu?) e tràsiri 'entrare' (A trasi a machina pi piaciri?). Il parlante diglotta è quasi sempre ignaro di usare una struttura inesistente nell'italiano standard. Ne diventa consapevole, spesso con frustrazione, quando qualcuno fa notare che in italiano i verbi uscire e entrare si usano esclusivamente come intransitivi.

Torniamo alla glottodidattica. In assenza di istruzioni esplicite, l'insegnante potrebbe ricorrere alla iterata correzione degli errori commessi. Da un lato, è

\footnotetext{
${ }^{12}$ Per la Critical Period Hypothesis, v. Lenneberg, 1967 e, per una sintesi riguardante gli effetti sull'apprendimento di una LS, LighTBOwn e SPADA, 1999: 60-65.
} 
probabile che questo lascerebbe l'apprendente insoddisfatto e demotivato, perché lasciato all'oscuro delle cause degli errori. Dall'altro, rimangono piuttosto dubbi i vantaggi sulla rapidità della soluzione del problema, che persisterebbe probabilmente più a lungo e con seri rischi di fossilizzazione. Nell'insegnamento guidato sembra così utile, se non necessario, il cosiddetto noticing (SCHMIDT, 1990; YIP, 1994), che richiede insegnanti con un'attenta preparazione, in grado di trasmettere agli apprendenti la nozione di valenza (così come qualcuno dei numerosi problemi che la nozione cela ${ }^{13}$ ) in forme semplici ed efficaci.

Il quadro dei lavori dedicati dagli specialisti in glottodidattica all'uso della nozione di valenza non è tuttavia interamente negativo ${ }^{14}$. Esistono in questo senso apprezzabili iniziative, ad esempio quella dell'Università di Milano, che nel 2012 ha organizzato un convegno dal titolo 'Il ruolo della grammatica valenziale nell'insegnamento delle lingue straniere'. Nell'incontro si sono sottolineati gli sviluppi di questo paradigma che, come si legge nel programma, «si presta ad un ampio utilizzo in sede didattica e in particolare nell'insegnamento delle lingue straniere» ${ }^{15}$. I dati riportati nella Sezione 3 non sembrano però in linea con l'affermazione che, dalla Germania, «la grammatica valenziale si sia poi diffusa a livello internazionale, prima nell'insegnamento del tedesco come L2, successivamente nella didattica delle altre lingue» ${ }^{16}$. Un certo interesse l'hanno manifestato anche altri ricercatori italiani, fra i quali Francesco Sabatini ${ }^{17}$, anch'egli intervenuto al convegno milanese del 2012.

\section{Valenza e significato}

Ai fini della pratica didattica, bisognerebbe riflettere bene su definizioni della valenza basate sul significato. Nella citazione di ALLERTON (2006) fornita sopra, ad esempio, l'autore non fa menzione del significato. Altri, non la maggioranza, operano la stessa scelta, come fa ROSEN (1991: 138): «the valence [...] registers a predicate's combinatory properties».

Alla voce 'Argument' di Wikipedia, strumento molto utilizzato dagli studenti e spesso specchio dell'opinione più diffusa ${ }^{18}$, si legge che «an argument is an

\footnotetext{
${ }^{13}$ «In Harris [Zellig Sabbettai] appaiono già, seppur trattati per accenni, i due problemi principali che pone la tematica delle reggenze: la difficoltà di distinguere attanti da circostanziali, e la questione dell'ellissi (o cancellazione) di argomenti obbligatori» (RENZI e ELIA 1997: 114).

${ }^{14}$ L'autore ringrazia Alberto Bramati per le informazioni gentilmente messe a disposizione.

${ }^{15}$ Dal sito web: http://www.medialin.unimi.it/files/programma_Valenza.pdf.

${ }^{16} \mathrm{Dal}$ sito web: http://www.laricerca.loescher.it/lingua-italiana/333-la-chimica-della-frase.html. La Germania è il paese in cui «[la grammatica valenziale] è nota col nome di "grammatica della dipendenza"» (ibid.).

${ }^{17} \mathrm{Si}$ veda SABATINI et alii, 2011.

${ }^{18}$ Quanto al valore di Wikipedia, istruttivo può essere un paragone tra 'unaccusative verb' e 'verbo inaccusativo' (12 giugno 2015). A fronte della definizione su basi sintattiche di David Perlmutter, che ha introdotto l'idea (v. ZAENEN, 2006), il testo inglese è tutto sbilanciato verso una definizione di natura semantica: «an unaccusative verb is an intransitive verb whose syntactic argument is not a semantic agent [...] an unaccusative verb's subject is semantically similar to the direct object of a transitive verb». Il testo italiano (alla voce 'Frase inaccusativa') contiene anche nozioni semantiche, ma significativamente recita: «la principale differenza tra verbi inaccusativi e verbi intransitivi [inergativi] è che, mentre il soggetto dei verbi inaccusativi presenta anche le proprietà dell'oggetto diretto, il soggetto dei verbi intransitivi non ha tali proprietà».
} 
expression that helps complete the meaning of a predicate» ${ }^{19}$. L'implicito di questa definizione di 'argomento' è che si attribuisce al predicato un significato preesistente alla combinazione sintattica. Anche se parecchio diffusa, riteniamo che una tale caratterizzazione della valenza vada incontro a problemi insuperabili. Se con 'significato' si intende legare la proposizione (realtà linguistica) e il mondo fenomenico (realtà extra-linguistica), una questione che rientra nelle delicate sfere della filosofia del linguaggio e dell'arbitrarietà saussuriana ${ }^{20}$, è facile mostrare che il legame è problematico ${ }^{21}$. Date le nostre conoscenze del mondo, ad esempio, i verbi abitare e andare dovrebbero entrambi aggregare almeno due argomenti, uno dei quali con il ruolo semantico di Locativo. Ma la sintassi non tiene conto di ciò ${ }^{22}$ : abitare il Locativo lo impone $(*$ Leo abita), mentre con andare è opzionale (Leo va).

Bene sarebbe, in linea con gli insegnamenti di Saussure ${ }^{23}$, un'istruzione nella quale si trasmettesse al discente l'idea che il significato si forma in funzione delle combinazioni sintattiche (v. LA FAUCI, 2011: 25-36). Gli esempi sono innumerevoli, con un posto di rilievo occupato da variazioni tra significato letterale o metaforico. In questa sede se ne propone soltanto uno, illustrabile con sfoderare la spada vs. sfoderare un sorriso, in cui al variare dell'Oggetto diretto, e delle funzioni solo argomentali (nel caso di la spada) o anche predicative (nel caso di un sorriso $)^{24}$ che questo assume, varia anche il significato.

\section{Conclusione}

L'uso consapevole e strutturato della valenza nell'insegnamento e nell' apprendimento della LS è il tema in breve affrontato in queste pagine. Per le considerazioni svolte, il quadro che emerge è quello di una sostanziale sottovalutazione in letteratura delle potenzialità della nozione in glottodidattica.

\footnotetext{
${ }^{19}$ Dal sito web: http://en.wikipedia.org/wiki/Argument_(linguistics).

${ }^{20}$ Almeno per coloro che vedono nell'arbitrarietà una «relationship between linguistic signs and the extralinguistic reality» (BUSSMANN, 1996: 32).

${ }^{21}$ CRUSE (2000 : 284-285) mostra tale problematicità per coppie di quasi-sinonimi.

${ }^{22}$ In questo senso, suscitano ammirazione e curiosità le parole di Samuel Taylor Coleridge scritte nel 1827 (per alcuni nel 1826), riportate da SAEED (1997: 336): «It is the fundamental mistake of grammarians and writers on the philosophy of grammar and language to suppose that words and their syntaxis are the immediate representatives of things. Words correspond to thoughts, and the legitimate order and connection of words to the laws of thinking and to the acts and affections of the thinker's mind».

${ }^{23} \mathrm{Si}$ veda Derossi (1965 : 89-90): «Il postulato basilare, del quale bisogna sin dall'inizio appropriarsi saldamente, e avere sempre di mira in seguito, se non si voglia scivolare inavvertitamente - com'è in ogni momento estremamente facile - fuori dalla più genuina concezione saussuriana, è il seguente: ciò che viene prima, nell'ambito linguistico, consiste nel legame fra le cose, non nelle cose stesse» (corsivi nell'originale). Il testo, tuttavia, può indurre a pensare alle «cose» come ontologicamente precedenti (v. LA FAUCI, 2011: 15-16).

${ }^{24}$ Interessante, anche nella prospettiva della glottodidattica, lo spazio creativo lasciato al parlante nel caso di costrutti con verbo supporto. Per via del contributo nullo del verbo supporto nella determinazione dei ruoli semantici ( $m a$ non in altri tipi di significato, per es. nelle variazioni di registro), la scelta dei sinonimi può essere ampia. Nel caso del nome predicativo sorriso, valga qui, insieme ai noti fare, dispensare, elargire e scoccare, l'esempio Martinelli inalbera un sorriso a trentadue denti, tratto da Il Fatto Quotidiano del 25 settembre 2011, pag. 3.
} 
Esistono apprezzabili tentativi di inserimento, ad esempio in Germania e in Italia, ma il tema stenta a entrare con forza e vitalità nel dibattito teorico e, conseguentemente, nella quotidiana pratica degli insegnanti di LS, a detrimento, pensiamo, dell'efficacia didattica.

\section{RIFERIMENTI BIBLIOGRAFICI}

ABbagnano Nicola (1971), Dizionario di filosofia, Torino, Utet.

AllerTON David J. (2006), 'Valency grammar', in: Brown (2006), vol. XIII, p. 301-314.

BETTONI Camilla (2001), Imparare un'altra lingua, Bari, Laterza.

BRAIDI, Susan M. (1999), The Acquisition of Second Language Syntax, London / New York, Arnold.

BROWN Keith (2006, chief editor), Encyclopedia of Language and Linguistics, $2^{\text {nd }}$ edition, Oxford, Elsevier.

BuSSMANN Hadumod (1996), Dictionary of Languageand Linguistics [translated and edited by G. Trauth and K. Kazzazi], New York / London, Routledge.

COMRIE Bernard (1981), Language Universals and Linguistic Typology, Chicago, The University of Chicago Press.

CRUSE Alan (2000), Meaning in Language, Oxford, Oxford University Press.

DAVIES William e Stanley DUBINSKY (1991), On representing and constraining lexical government of syntactic structures, in: Linguistics 29, pp. 1011-1051.

DEROSSI Giorgio (1965), Segno e struttura linguistici nel pensiero di Ferdinand de Saussure, Trieste, Del Bianco Editore.

Ellis Rod (1994), The Study of Second Language Acquisition. Oxford, Oxford University Press.

FRIED Vilém (1972), The Prague School of Linguistics and Language Teaching, Oxford, Oxford University Press.

HAJIČOVÁ Eva (2006), Prague School, in: Brown (2006), vol. X, pp. 62-67.

JOHNSON Keith e JOHNSON Helen (1999), Encyclopedic Dictionary of Applied Linguistics, Oxford / Malden (MA), Blackwell.

LADO Robert (1957), Linguistics Across Cultures, Ann Arbor, University of Michigan Press.

LA FAUCI Nunzio (2011), Relazioni e differenze. Questioni di linguistica razionale, Palermo, Sellerio.

LARSEN-FREEMAN Diane e LONG Michael H. (2014 (1991)), An Introduction to Second Language Acquisition Research, New York, Routledge.

LENNEBERG Eric H. (1967), Biological Foundations of Language. New York, Wiley.

Lightbown Patsy M. e SPADA Nina (1999), How Languages are Learned, Oxford, Oxford University Press.

Mitchell Rosamond e MYLES Florence (1998), Second Language Learning Theories, London, Arnold.

RENZI Lorenzo; ELIA Annibale (1997), Per un vocabolario delle reggenze, in: SLI, Lessico e grammatica. Teorie linguistiche e applicazioni lessicografiche, Roma, Bulzoni, pp. 113-129. 
RIEGER Marie A. (2003), I dizionari della valenza verbale e l'insegnamento del tedesco come lingua straniera, Quaderni del Cirsil, 2, www.lingue.unibo.it/cirsil, pp. 1-27.

ROSEN Carol (1991), Relational Grammar: L2 learning and the components of L1 knowledge, in: HUEBNER Thom; FERGUSON Charles A. (a cura di), Crosscurrents in Second Language Acquisition and Linguistic Theories, Amsterdam / Philadelphia, John Benjamins, pp. 123-142.

SABAtini Francesco, Carmela CAmodeca e De SANTis Cristiana (2011), Sistema e testo. Dalla grammatica valenziale all'esperienza dei testi, Torino, Loescher.

SAEED John I. (1997), Semantics, Oxford, Blackwell.

SCHMIDT Richard W. (1990), The role of consciousness in second language learning, Applied Linguistics 11, pp. 129-158.

SELINKER Larry (1972), Interlanguage, in: ROBINETT B.W. e SCHACHTER J. (eds.), Second Language Learning, Ann Arbor, The University of Michigan Press, pp. 173-196.

TESNIÈRE Lucien (1959), Éléments de syntaxe structurale, Paris, Klincksieck.

VACHEK Josef (1972), The linguistic theory of the Prague School, in: Fried (1972), pp. 11-28.

YIP Virginia (1994), Grammatical consciousness-raising and learnability, in: OdLIN T. (ed.), Perspectives on Pedagogical Grammar, Cambridge, Cambridge University Press.

ZAENEN Annie (2006), 'Unaccusativity', in: Brown (2006), Volume XIII, pp. 217 224. 